\title{
Remote Monitoring System for Resistance Welder Machine
}

\author{
Hao Xu ${ }^{\text {a }}$, Zhonglin Gao ${ }^{b}$ Yangzhan $\mathrm{Yu}^{\mathrm{c}}$ and $\mathrm{Ge}$ Yin ${ }^{\mathrm{d}}$ \\ Tianjin 707 Hi-tech Co., Ltd, Tianjin 300402, China. \\ axh_20050@163.com, b13502051678@sina.cn, cyyz0208@126.com, dseamanyeen@163.com
}

\begin{abstract}
A system design for monitoring resistance welders remotely based on wireless communication and Internet. It combines field welder group-control system with remote communication technology and replaces the wired-base system with wireless network. The system follows the principle of modular and distributed design. The core module is a service-oriented communication module which transfers data between industrial fields and remote server.
\end{abstract}

Keywords: Resistance Welder, Wi-Fi; Modular Design, WCF, Remote Monitoring System.

\section{Introduction}

The resistance welder group-control system has now been widely used in automobile manufacturing industry. It provides an efficient way to centralized monitor and manage welding equipment. Most of these systems are based on industry field-bus which means that their communication is wired-based. [1] This kind of design powerful guarantees the real-time and reliability of communication sacrificing the mobility and installation complexity. [2] In recent years, with the development of advanced wireless technology such as LTE, LTE-A and WLAN-11, wireless technology has been applied in more and more areas. However, in the field of welding management, there are almost no corresponding application examples. So, we try to discuss its application and prospect in the welding group-control.

With wireless technology, the mobility of group-control system can be greatly improved since wireless network installation is easy and it requires less time. Another benefit is that the process of troubleshooting is much easier comparing to wire-based network. Considering the practical welding application, though the stability and reality may be decreased, the system can still meet the requirements for use.

To improve the application potential of system, we also extend the system to Internet application. A remote server is deployed to communicate with field server and provide data interface for remote applications. The whole system establishes a practical solution for resistance welder controlling including monitoring, troubleshooting, programming, etc.

\section{Field Server Structure}

\subsection{System Overview.}

The entire system is designed following to the principle of modularity. As shown below (figure 1), the system is modularized into 3 parts: field data collection module, service-oriented communication module, and user application module. Each part communicates with others following rigorous use of well-defined data interfaces. Both the field system and remote system provide welding monitoring function and data query function. The detailed function for each part will be shown in later sections. 


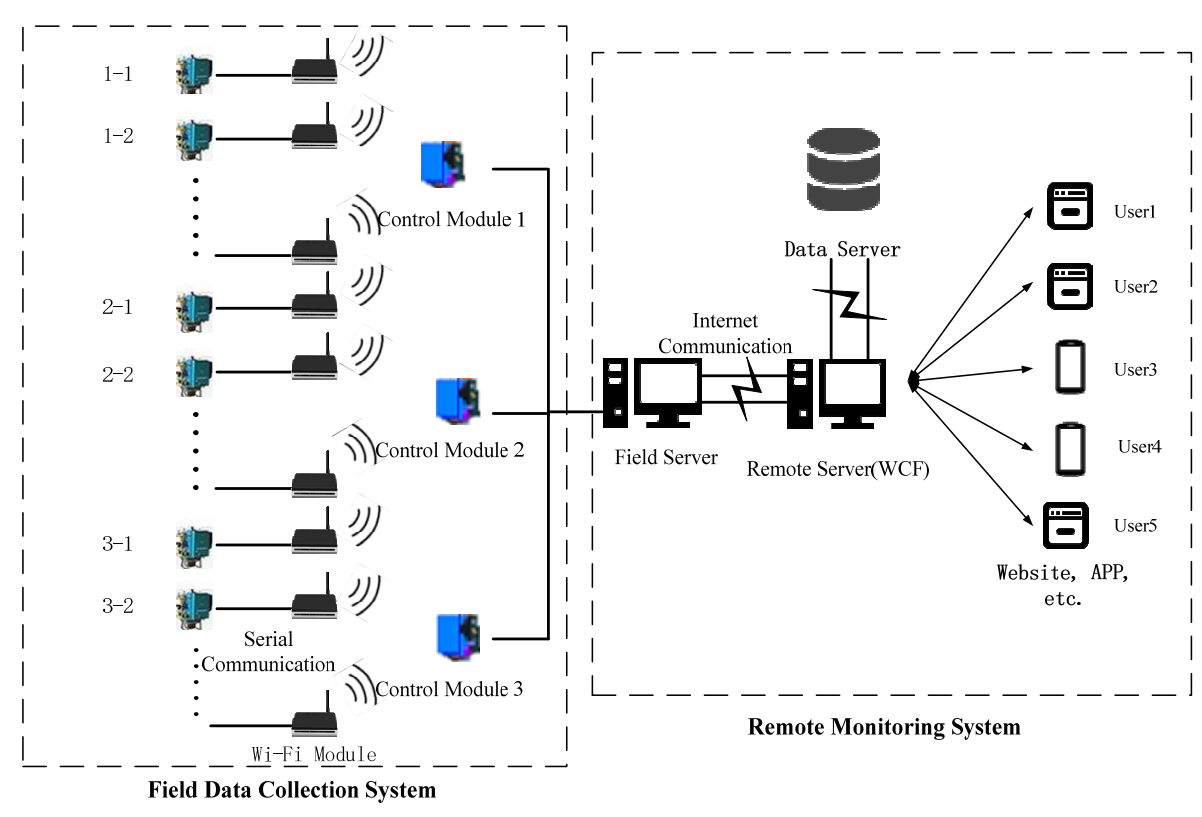

Figure 1. System Structure

\subsection{Field System.}

The field system adopts three-layer structure: welder machine - control modules (PLC) - field server. [1] The welder machine is connected to the Wi-Fi module to communicate with every control module. The wireless communication uses $802.11 \mathrm{~g}$ technology. As the middle layer of the system, PLC devices has two functions. The first one is collecting data including welding record, trouble alarm, welding standard, etc. PLC has been programmed to respond different of data, and then finally send to the PC server after processing. The other one is monitoring grid voltage and according to voltage situation to control welders' operation. Since resistance welder is high-power device, this function is very necessary and we have designed a specific algorithm to control the welding sequence. The detailed process is shown as figure 2. The filed server is an industrial personal computer which runs a MFC application to display the real time situation of all welders and provide interoperate for users. For remote monitoring, the server program must able to connect to the Internet and keep accessing the data interface on the remote server.

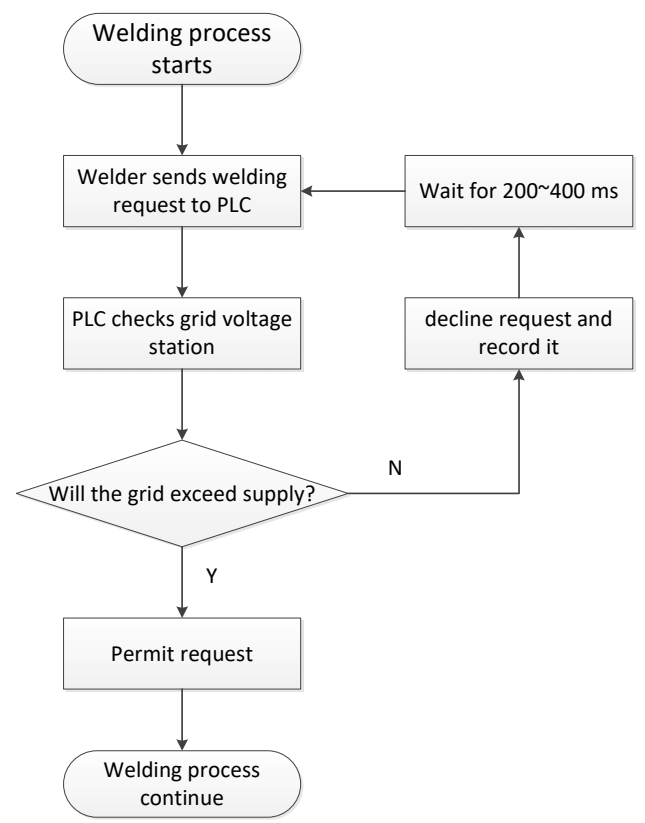

Figure 2. Grid Monitoring Logic 


\subsection{Wi-Fi Structure Design.}

As section 2.2 mentioned, the network mainly transmits two kinds of data in the system: one is the welding permit/decline command, the other is various data of welding situation. The second kind of data is at most transmitted once in each period of welding process (in most condition, it's usually longer than 1s) and the data length is no more than 80 bytes. The first kind, by contrast, is transmitted in a much higher frequency (about 3 5 times every second) and the data length is 20 bytes each. So, to guarantee that the system is reality, we choose the $802.11 \mathrm{~g}$ technology as the Wi-Fi standard which has a speed of $54 \mathrm{Mbps}$. After balancing the reality and cost, we have designed several options for different application cases covering from a few machines to hundreds of machines and the networking equipment includes AP, switches or even super AP. One of the most representative examples is the application in Shan Xi Heavy Duty Automobile's welding workshop which covers an area of 3 acres. To balance the reality of network and the cost and also avoid channel disturbance, we use 3 wireless access points, routers and adapters to establish the network. And it proves the system can successfully run and keep stable.

\section{Remote Monitoring System}

3.1 Communication Server.

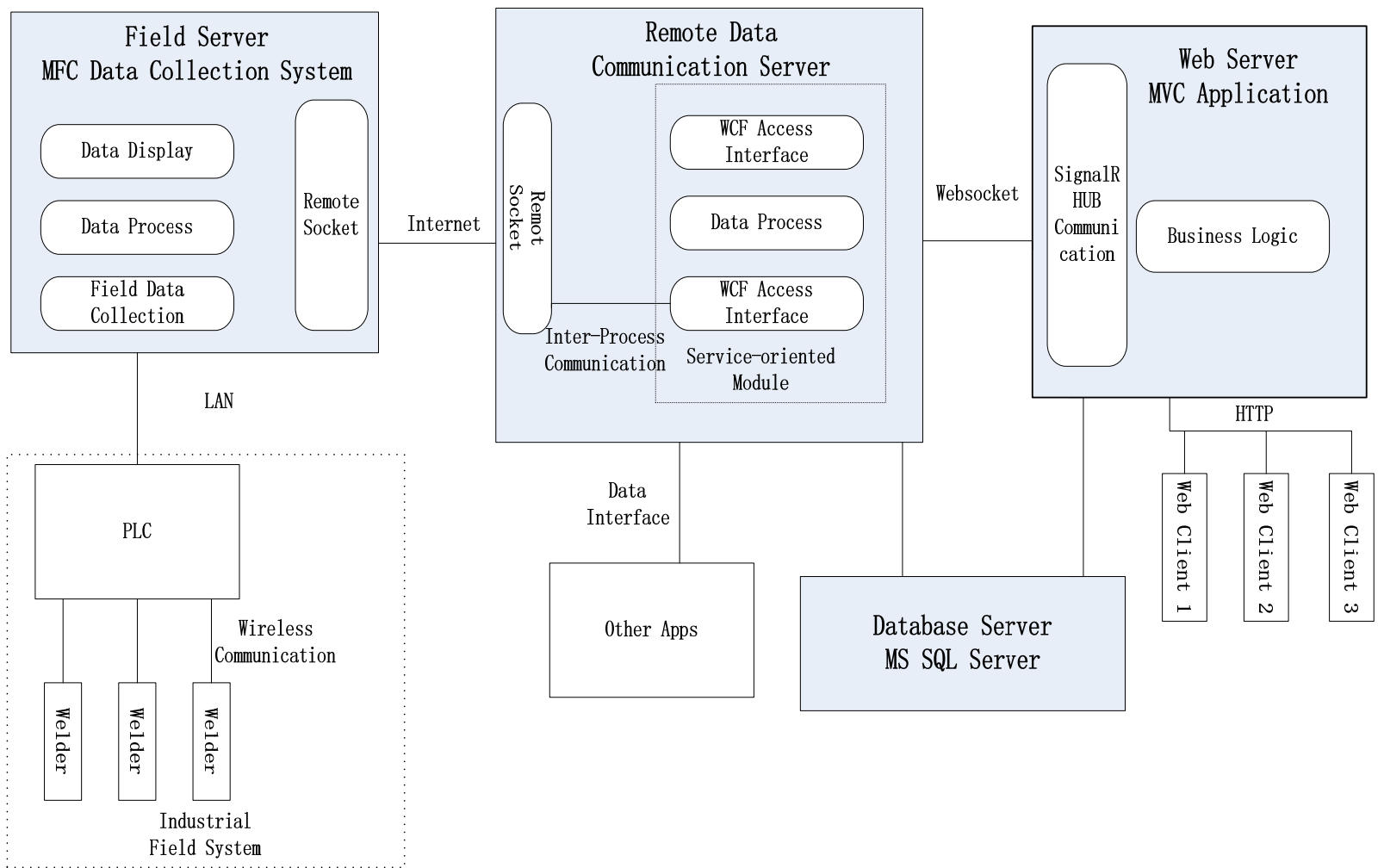

Figure 3. System Principle

In Figure 3, we deploy a WCF program on the remote server. The WCF program is a serviceoriented application which provides a link between the field and remote users. It has two series of data interface, one is used to accept the real-time data from field system, and the other one is open to remote users to achieve data. It also has the function of saving the welding record to database. The database provides query service of welding history for remote users.

\subsection{MVC Application}

To test the practical performance of this system, we also develop a Web MVC application based on $\mathrm{C \#}$ language. The website provides basic data query service and remote monitoring function. To make sure that the monitoring function is real-time, the website establishes a web-socket connection with the communication server based on SignalR library. [3] Except the web-socket way, other 
applications such as mobile app can also use the data interfaces of communication server to get realtime welding data in polling ways. Figure 4 are some screenshot samples of the website.

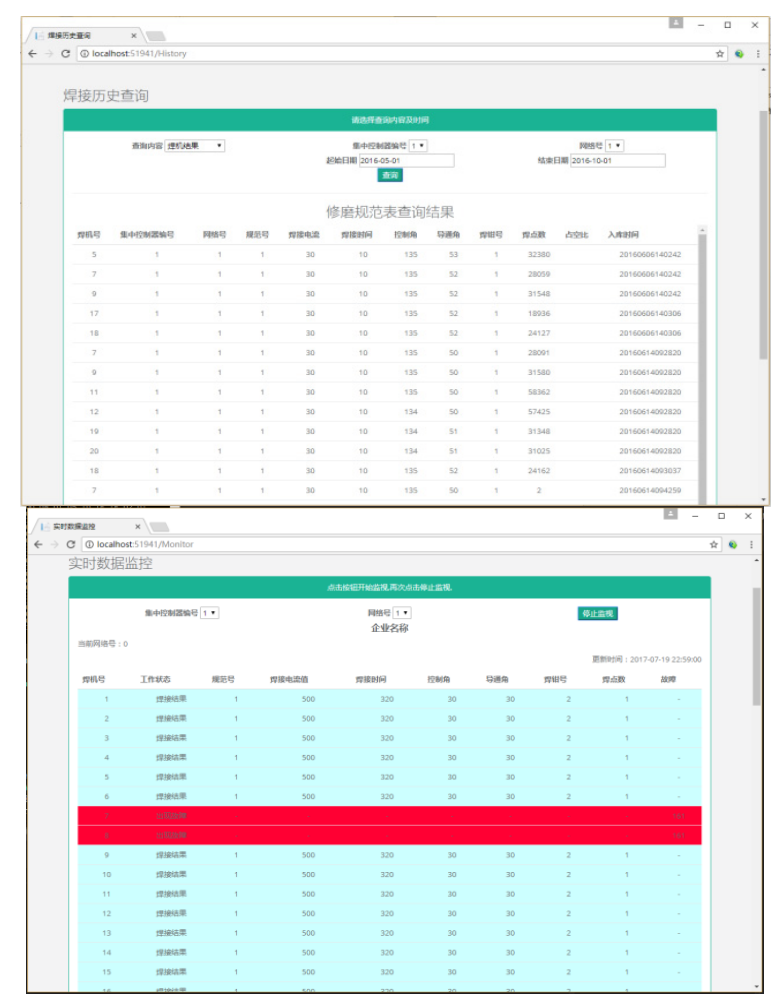

Figure 4. Left: Date Query Result, Right: Monitoring Display

\section{Summary}

Though advanced wireless technology has been widely used in many areas, it is still rarely used in resistance welding management and other automobile manufacturing area. Comparing to wirebased communication, its mobility, convenience and flexibility are much better, and also the troubleshooting becomes easier which is a big headache for wired one. Through this endeavor, we hope to promote the application of wireless communication and we are happily to see that our system has now been successfully applied in several companies such as Zhengzhou Nissan, Shan Xi Heavy Duty Automobile, FAW JiLin, etc.

\section{References}

[1]. Cheng Haihui, Yan Xuesen, Guo Jinhua, Yin Ge, Development of Networked Group Control System for Resistance Welding Machine [J]. Modern Electronic Technology, 2008, 07, 148-150.

[2]. Yan Dong, Han Eryang, Zheng Bo, Lu Qibing, Communication Technology in Welding Production Monitoring [J]. Electric Welder, 2015, 04, 17-20.

[3]. Li Yan. Realization of Real-time Web Function Based on ASP.NET SignalR[J]. Computer Knowledge and Technology, 2016, 12(24).

[4]. Yin Ting, Zhao Sijia. Research on AJAX Website Design Pattern Based on jQuery Framework[J]. Journal of Hunan Ecology, 2010, 16(3):1-4. 